\title{
Age Estimation by Modified Demirjian’s Method in a Hospital- Based Population: A Radiographic Study
}

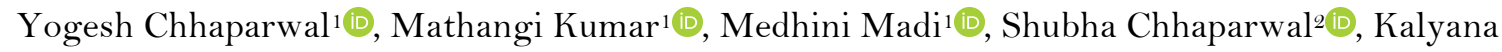 \\ Chakravarthy Pentapatis (1)
}

\begin{abstract}
'Department of Oral Medicine \& Radiology, Manipal College of Dental Sciences, Manipal, Manipal Academy of Higher Education, Manipal, Karnataka, India.

${ }^{2}$ Department of Conservative Dentistry and Endodontics, Manipal College of Dental Sciences, Manipal, Manipal Academy of Higher Education, Manipal, Karnataka, India.

${ }^{3}$ Department of Public Health Dentistry, Manipal College of Dental Sciences, Manipal, Manipal Academy of Higher Education, Manipal, Karnataka, India.
\end{abstract}

Correspondence: Mathangi Kumar, Department of Oral Medicine \& Radiology, Manipal College of Dental Sciences, Manipal, Manipal Academy of Higher Education, Manipal, 576104, Karnataka, India. E-mail: madhukumar4u@gmail.com, mathangi.kumar@manipal.edu

Academic Editor: Alessandro Leite Cavalcanti

Received: 17 June 2020 / Review: 14 December 2020 / Accepted: 22 December 2020

How to cite: Chhaparwal Y, Kumar M, Madi M, Chhaparwal S, Pentapati KC. Age estimation by modified Demirjian's method in a hospital-based population: a radiographic study. Pesqui Bras Odontopediatria Clín Integr. 2021; $21: e 0137$. https://doi.org/10.1590/pboci.2021.072

\begin{abstract}
Objective: To test the modified Demirjian's method of dental age estimation on the South Indian population and correlate it with the patient's chronological age. Material and Methods: This retrospective radiographic study was performed on digital panoramic radiographs taken during a one-year duration. Radiographs of patients in the age range from 8 to 18 years were included in the study. The radiographs were examined and according to the stage of the crown and root status of the left side mandibular teeth. The stage of calcification was observed for eight teeth in the mandibular arch and was recorded in a specially designed proforma. Each rated tooth with stage was converted into a score using a conversion table specified for girls and boys. Results: 224 digital panoramic radiographs were retrieved from the radiologic database. Out of total study sample, $49.5 \%(n=111)$ were boys and $50.5 \%(n=113)$. The mean chronological and estimated dental age using dental maturity scores resulted in an overestimation of 3 years and eight months. Also, it was noted that there was a strong positive association between chronological (real) and dental age. Conclusion: It was observed that there was a strong correlation between chronological and dental age. A new formula for determining the chronological age from the estimated dental age was derived. Further studies on a larger population may prove the reliability of this age estimation method.
\end{abstract}

Keywords: Forensic Dentistry; Age Determination by Teeth; Radiography, Panoramic. 


\section{Introduction}

Age estimation may not always be important only in crime scenes, natural disasters, and accidents. Its applicability is tremendous in rural areas where the awareness of childbirth registration and recordkeeping is bare minimal. Apart from this, instances of serious crimes committed by juveniles, intentional manipulation of the definite date of birth for procuring jobs, selections in sports and higher education have increased the need for accurate chronological age estimation methods for legal considerations and forensic science [1].

Dental age estimation can be the most practical and doable option when correlated with the patient's chronological age. Numerous methods have been used to estimate dental age. Demirjian et al. [2] put out Demirjian's method of age estimation in the year 1973, which subsequently became the most popularly applied method for dental age estimation [2,3]. This method originally used seven teeth of the mandibular left quadrant. The downside of this technique was that it did not take the third molar into consideration. This could be because the third molar had a high propensity of being congenitally missing and the third molars also had a wide variation in their development. Few researchers altered and revised the original Demirjian's method using the data collected for their population. Demirjian too published a modified version of his method to overcome the deficiencies of his original technique. He added the staging that took the third molars into consideration [4].

Hence, this study was conducted to test the modified Demirjian's method of dental age estimation on the South Indian population and correlate it with the patient's chronological age.

\section{Material and Methods}

\section{Study Design}

This retrospective radiographic study was performed on digital panoramic radiographs of patients taken during a one-year duration. Radiographs were retrieved from the archives of Dental Radiology department. A person who was not a part of the study anonymised all the radiographs.

Radiographs of patients in the age range from 8 to 18 years were included in the study. Radiographs of patients belonging to other ethnicities other than Indian origin and radiographs with movement artefacts and poor image quality obscuring the area of interest were excluded from the study. The radiographs were examined and according to the stage of the crown and root status of the left side mandibular teeth. Following this, the dental age estimation was done according to the Modified Demirjian's method. The stage of calcification was observed for eight teeth in the mandibular arch and was recorded in a specially designed proforma.

\section{Digital Panoramic Radiographs}

The panoramic radiographs were obtained from Planmeca ProMax 2D S3 machine (Planmeca OY, Finland). Radiographs, which had missing third molar, multiple missing teeth, unilateral hypodontia, were excluded from the study. Standard protocols for imaging were followed according to the ALARA principle [5]. The images were viewed on a personal computer under standard brightness for appropriate interpretation.

Dental maturity scores were calculated by scoring the eight permanent left side mandibular teeth. The teeth that were considered were incisors, canines, premolars and molars. Based on the stage of tooth development, the scores were assigned according to the ratings proposed by Chaillet and Demirjian [6]. These data were recorded in a specially designed proforma. Two oral radiologists independently viewed all the radiographs and performed the scoring. The dental maturity scores were obtained by summing up the scores of 
left mandibular teeth. Each rated tooth with stage was converted into a score using a conversion table specified for girls and boys [6]. The radiographs were completely anonymised and hence the chronological age was unidentifiable.

To assess the intra and inter-observer reliability, the two observers repeated the scoring for $10 \%$ of the samples at an interval of two weeks. After this, the radiographs were decoded, and the chronological age was retrieved from the patient case record. The chronological age was taken as the age at which the radiographic examination was performed.

Correlation between Chronological (Real) and Estimated Dental Age

Linear regression analysis was used to produce new maturity scores for dental age. The following formula was used for calculating chronological age from the estimated dental age:

$$
\begin{gathered}
\text { Age }(\text { Males })=\text { Chronological age }+3.091(95 \text { th Dental age }) \\
\text { Age }(\text { Females })=\text { Chronological age }+3.527(95 \text { th Dental age })
\end{gathered}
$$

Data Analysis

The dental maturity scores, the chronological age and the predicted age of all patients were entered in Microsoft Excel spreadsheet and statistical analysis was performed using IBM SPSS Statistics for Windows, Version 21.o. (IBM Corp., Armonk, NY, USA). Inter and intra-observer agreement was determined by paired Student's t-test, and Pearson correlation between chronological age and dental age was performed. Linear regression analysis was done for obtaining the new maturity scores for dental age of patients. A p-value less than 0.001 was considered significant.

\section{Ethical Clearance}

Institutional ethical committee clearance was obtained to perform this study (Protocol No. 645/2016).

\section{Results}

Two hundred and twenty-four digital panoramic radiographs were retrieved from the radiologic database (February 2015 to February 2016). Out of the total study sample, $49.5 \%(\mathrm{n}=111)$ were boys and $50.5 \%(n=113)$ were girls with an age range from 8 to 18 years (Table 1$)$.

Table 1. Distribution of participants according to age and sex.

\begin{tabular}{cccc}
\hline Age (in Years) & Male & Female & Total \\
& N & N & N \\
\hline 8 & 6 & 3 & 9 \\
9 & 7 & 7 & 14 \\
10 & 8 & 9 & 17 \\
11 & 9 & 6 & 15 \\
12 & 9 & 8 & 17 \\
13 & 3 & 4 & 7 \\
14 & 11 & 7 & 18 \\
15 & 10 & 9 & 19 \\
16 & 8 & 11 & 19 \\
17 & 14 & 18 & 32 \\
18 & 26 & 31 & 57 \\
\hline
\end{tabular}


The inter and intra-observer's error was calculated and there were no significant differences found. The inter and intra-observer consistency was 99\%. The mean chronological and estimated dental age using dental maturity scores resulted in overestimation of 3 years 8 months (Table 2 ).

Table 2. Applicability of modified Demirjian's method.

\begin{tabular}{lcccccccc}
\hline \multirow{2}{*}{ Gender } & \multicolumn{2}{c}{ Predicted Age 95 } & \multicolumn{2}{c}{ Chronological Age } & \multicolumn{2}{c}{ Difference } & \multicolumn{2}{c}{ T } \\
& Mean & SD & Mean & SD & Mean & SD & \\
\hline Male & 10.36 & 0.96 & 14.18 & 3.35 & -3.82 & 2.54 & -15.84 & $<0.001$ \\
Female & 10.39 & 0.72 & 14.73 & 3.23 & -4.33 & 2.71 & -17.01 & $<0.001$ \\
\hline
\end{tabular}

Correlation between Chronological (real) and Estimated Dental Age

Age of males: Chronological age $=-17.847+3.091$ (95th Dental age), Male $\mathrm{R}^{2}=0.782$

Age of females: Chronological age $=-21.924+3.527$ (95th Dental age), Female $\mathrm{R}^{2}=0.61$

Also, it was noted that there was a strong positive association between chronological (real) and dental age (Figure 1).

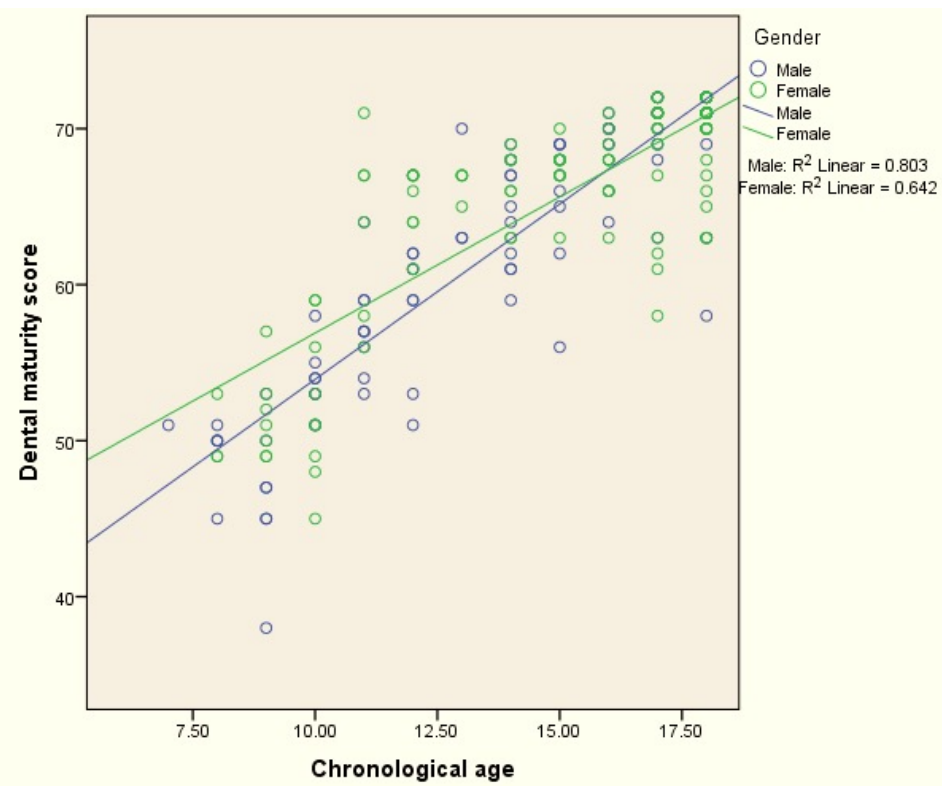

Figure 1. Correlation between chronological (real) age and dental maturity score.

\section{Discussion}

Age estimation is a vital part of forensic sciences. It is of paramount importance in medico-legal disputes pertaining to the age of an individual. Most of the traditional age estimation methods are invasive in nature, requiring expensive armamentarium and instrumentation. Moreover, the procedures might be very lengthy and might require experienced pathologists to deduce the individual's age. Forensic Odontology and its age estimation methods have become increasingly popular. Since age estimation in children and adolescents needs to be exact, accurate and dependable from a legal perspective, the dental surgeon is most likely to be called for an expert witness in legal room proceedings in such cases [7,8].

Several methods have been formulated for age estimation. One of the most extensively used and studied is Demirjian's method, which was introduced in 1973 that was based on a French-Canadian population [9]. This method uses a panoramic radiograph and inspects the seven left permanent mandibular teeth for the 
estimation of the extent of mineralized dental tissues and the shape of the pulp chamber. This method gained popularity since tooth maturation was a better indicator of age than tooth eruption. Tooth eruption is influenced by several other external factors like infections, impactions, ankylosis, crowding and extractions. The original method also excluded the third molars due to inconsistencies in their development, eruption and anatomy [7]. A few years later, after the original method was published, Demirjian and Goldstein revised this method to overcome limitations in its applications. The modified Demirjian stages of tooth development used in the present study were intended to include the third molars. The third molars essentially are the only reliable parameter for age estimation in the age group of 16-23 years [4]. Chaillet and Demirjian made another significant modification to the original Demirjian method. They included two more stages: the stage of nonformation of tooth, namely "Stage 0", and the stage of crypt development, i.e., "Stage 1" [7].

Research has shown substantial differences in the estimated means of the dental age and chronological age when this method and the reference data provided by the method are applied to other populations [9]. Since the teeth development schedule can vary between populations, age estimation must be made in the geographic regions presenting the same characteristics [10].

In the present study, modified Demirjian's method was applied to the Coastal Karnataka population. Inter and intra-observer errors were calculated and there were no significant differences found. Inter- and intraobserver consistency was rated at $99 \%$. A strong positive association was found between the chronological (real) age and the dental age. The results were in accordance with the literature [4,11,12]. An age estimation method can be considered efficient and effective if it has higher accuracy and precision with relatively low bias. Precision can be partially credited to the inter and intra-observer reliability. The cause for an increase in the inter and intra-observer reliability in modified Demirjian's method could be ascribed to the reduced stages of grading development. Additionally, in modified Demirjian's method, each stage is clearly described and is complete. Further, there is no requirement for measurements or assessment of the relative progression of a stage. Moreover, the rules for decision-making to determine the stages are clearly delineated.

Ayranci et al. [10] evaluated the modified Demirjian's method on Turkish children and young adults. It was reported that in the Turkish children and young adults, the mean ages at A, C, D, E, F1, G, G1 developmental stage were lower for males than for females and B and $\mathrm{F}$ were lower for females than males. Goya et al. [13] estimated the dental age in Japanese children using a modified Demirjian method. Blenkin and Evans [14] tested the Demirjian system's applicability on a sample of the Sydney child population. All three studies reported an overestimation of dental age in comparison to chronological age. The findings were in concordance with the present study that also reported an overestimation of dental age.

Dehankar et al. [7], Pratyusha et al. [8], and Priyanka et al. [11] performed the age estimation analysis by modified Demirjian's method in different geographical provinces of India. Dehankar et al. [7] reported that 15 subjects $(30.6 \%)$ had an error in age estimation less than 1 year and 12 subjects (24.5\%) had an error of more than 2 years. It was also reported that they found a remarkable difference between chronological and dental age in the lower age groups (12-14 years), but, as the age increased (around 16-18 years), the variation narrowed down. The dental age was overestimated by Demirjian's method with a mean difference of 0.69 for girls and 0.23 for boys by Pratyusha et al. [8]. According to Priyanka et al. [11], dental age was found to be overestimated by +1.04 years with chronological Age. In the present study, the comparison between mean chronological age and the estimated dental age using dental maturity scores resulted in an overestimation of 3 years and eight months. 
Contrasting the present study results, Lee et al. [4] tested the validity of Demirjian's and modified Demirjian's methods in age estimation for Korean juveniles and adolescents. Demirjian's revised method was reported to overestimated age by 0.002 years for males and 0.227 years for females. The dental age showed no statistically significant difference from chronological age in males. Whereas in females, there was a significant difference. In contrast to the present study's findings, Mohammed et al. [15] found that Demirjian's modified method underestimated males' mean age by 0.8 years and the females by 0.5 years and showed that females mature earlier than males. They attributed this result to the possibility of low X-ray standards and socioeconomic status lower than that of the reference population.

Most of the studies done among Indian population have shown overestimations ranging from 2 months to over three years. Hence, an adaptation of the modified Demirjian's method to suit the local population is crucial. The population of a specific geographic region will be having a genetic make-up dependant on their ethnicity. They will be socioeconomically very different from the world population taken as a whole. Nutritional and dietary habits can also have a significant impact on skeletal and dental maturity [7].

Modified Demirjian's method is highly subjective. Differences in attributing even one stage higher or lower than the actual attributable stage could significantly impact the individual's dental age. To avoid these errors, the observers must be adequately trained to make accurate visual discrimination and judgement of the tooth development stages. However, we must also realize that no single age estimation method can accurately and flawlessly measure the individuals' exact age since every individuals' development may vary naturally. Repetitive measurements and measuring the age with more than one age estimation method would probably yield much closer and accurate results [11].

Hence, in this study, the modified Demirjian method was applied only to a hospital-based population for dental age estimation in the present study. Based on these observations, further studies with a larger population-based sample should be employed to obtain a reliable estimation of age.

\section{Conclusion}

In the present study, the modified Demirjian method of age estimation was applied on a hospital-based population. It was observed that there was a strong correlation between chronological and dental age. A new formula for determining the chronological age from the estimated dental age was derived.

\section{Authors' Contributions}

\begin{tabular}{|c|c|c|}
\hline YC & https://orcid.org/0000-0003-0182-3183 & $\begin{array}{l}\text { Conceptualization, Methodology, Formal Analysis, Investigation, Resources, Data Curation, } \\
\text { Writing - Original Draft and Writing - Review and Editing. }\end{array}$ \\
\hline MK & https://orcid.org/0000-0003-1245-2314 & $\begin{array}{l}\text { Conceptualization, Methodology, Formal Analysis, Investigation, Resources, Data Curation, } \\
\text { Writing - Original Draft and Writing - Review and Editing. }\end{array}$ \\
\hline MM & https://orcid.org/0000-0002-5484-5960 & Validation, Resources, Writing - Original Draft and Writing - Review and Editing. \\
\hline $\mathrm{SC}$ & https://orcid.org/0000-0002-0129-8553 & Methodology and Writing - Review and Editing. \\
\hline КСР & https://orcid.org/0000-0002-5462-5677 & Methodology, Validation, Writing - Review and Editing and Supervision. \\
\hline
\end{tabular}

\section{Financial Support}

None.

\section{Conflict of Interest}

The authors declare no conflicts of interest. 


\section{Data Availability}

The data used to support the findings of this study can be made available upon request to the corresponding author

\section{References}

[1] Jain V, Kapoor P, Miglani R. Demirjian approach of dental age estimation: Abridged for operator ease. J Forensic Dent Sci 2016; 8(3):177. https://doi.org/10.4103/0975-1475.195103

[2] Demirjian A, Goldstein H, Tanner JM. A new system of dental age assessment. Hum Biol 1973; 45(2):211-27.

[3] Demirjian A, Goldstein H. New systems for dental maturity based on seven and four teeth. Ann Hum Biol 1976; 3(5):411-2 1. https://doi.org/10.1080/03014467600001671

[4] Lee SS, Kim D, Lee S, Lee UY, Seo JS, Ahn YW, et al. Validity of Demirjian's and modified Demirjian's methods in age estimation for Korean juveniles and adolescents. Forensic Sci Int 2011; 211(1-3):41-6. https://doi.org/10.1016/j.forsciint.2011.04.011

[5] American Dental Association Council on Scientific Affairs. The use of dental radiographs: update and recommendations. J Am Dent Assoc 2006; 137(9):1304-12.

[6] Chaillet N, Demirjian A. Dental maturity in South France: A comparison between Demirjian's method and polynomial functions. J Forensic Sci 2004; 49(5):1059-66.

[7] Dehankar N, Naidu GS, Makkad RS, Nagi R, Jain S, Deshmukh U. Validity of Demirjian 8-teeth method for age estimation by orthopantomogram - a prospective study. J Indian Acad Oral Med Radiol 2018; 30(2):148-52. https://doi.org/10.4103/jiaomr.jiaomr_42_18

[8] Pratyusha K, Prasad MG, Radhakrishna AN. Applicability of Demirjian's method and modified Cameriere's methods for dental age assessment in children. J Clin Diagn Res 2017; 11(2):ZC40-ZC43. https://doi.org/10.7860/JCDR/2017/21474.9342

[9] Chiam S, Blenkin M, Taylor J, Moananui R Te, Blenkin M, Taylor J, et al. Validity of a modified Demirjian system based on an Australian dataset - simple maturity score in age estimation. Aust J Forensic Sci 2016; 48(5):1-12. https://doi.org/10.1080/00450618.2015.1107133

[10] Ayranci F, Omezli MM, Sivrikaya EC, Cezairli B, Eroglu A. Age estimation and its adaptation of the Turkish children using modified demirjian's method. MOJ Anat Physiol 2017; 3(6):174-7. https://doi.org/10.15406/mojap.2017.03.00113

[11] Priyanka M, Kiran G, Poornima R, Shravani R, Shobhita KC, Preethi P. Age Estimation by Modified Demirjian's method in children and adolescents of Hyderabad population - A panoramic study. J Orofac Sci 2018; 10(2):63-8. https://doi.org/10.4103/jofs.jofs_61_18

[12] Bijjaragi SC, Sangle VA, Fk S, Patil VS, Sr AR. Age estimation by modified Demirjian's method (2004) and its applicability in Tibetan young adults: A digital panoramic study. J Oral Maxillofac Pathol 2015; 19(1):100-5. https://doi.org/10.4103/0973-029X.157223

[13] Goya HA, Satake T, Maeda T, Tanaka S, Akimoto Y. Dental age in Japanese children using a modified Demirjian method. Pediatr Dent J 2009; 19(1):82-8. https://doi.org/10.1016/S0917-2394(09)70157-0

[14] Blenkin MRB, Evans W. Age Estimation from the teeth using a modified Demirjian System. J Forensic Sci 2010; 55(6):1504-8. https://doi.org/10.1111/j.1556-4029.2010.01491.x

[15] Mohammed RB, Koganti R, Kalyan SV, Tircouveluri S, Singh JR, Srinivasulu E. Digital radiographic evaluation of mandibular third molar for age estimation in young adults and adolescents of South Indian population using modified Demirjian’s method. J Forensic Dent Sci 2014; 6(3):191-6. https://doi.org/10.4103/0975-1475.137068 